\title{
Good Water Absorption and Anti-adhesion Properties of Designed Extra Thin PVA Foam Membranes with Fully Open-cell Microstructures Derived from a Super Clean Air-Foaming Process with Active Molecules for Minimally Invasive Surgery
}

\author{
Ching Cheng Huang*1,2 and Meng Jen Yang ${ }^{2}$ \\ ${ }^{1}$ Department of Biomedical Engineering, Ming-Chuan University, Taoyuan, Taiwan \\ ${ }^{2}$ PARSD Biomedical Material Research Center, Jiangsu
}

*Corresponding author: Ching Cheng Huang, Department of Biomedical Engineering, Ming-Chuan University, Taoyuan, Taiwan

ARTICLE INFO

Received: 蔧 February 19, 2019

Published: 蔧 February 27, 2019

\section{ABSTRACT}

Abbreviations: PVA: Polyvinyl Alcohol; ETPVAFM: Extra Thin Polyvinyl Alcohol Foam Membranes; MIS: Minimally Invasive Surgery; NPWT: Negative Pressure Wound Therapy

Citation: C-C Huang, Meng Jen Y. Good Water Absorption and Anti-adhesion Properties of Designed Extra Thin PVA Foam Membranes with Fully Open-cell Microstructures Derived from a Super Clean Air-Foaming Process with Active Molecules for Minimally Invasive Surgery. Biomed J Sci \& Tech Res 15(2)2019. BJSTR. MS.ID.002672.

\section{Introduction}

The design of medical devices could be applied and developed for new microscopic surgical procedures instead of the traditional surgical procedures. For the design of medical devices, selections of suitable materials for biomedical applications such as polymethacrylate, polyester, polynorbornene, and polymeric resins could be substantially considered and further employed [1-4]. The surface modification could be considered to change the surface microenvironment of materials for specific need [5-6]. Furthermore, the preclinical evaluations of materials and medical devices by using Polyvinyl Alcohol (PVA) foam must be considered for the application and design [7]. In this study, a new anti-adhesion Extra Thin Polyvinyl Alcohol Foam Membranes (ETPVAFM) derived from a super clean air-foaming process was designed and prepared for Minimally Invasive Surgery (MIS). Preclinical evaluation of designed anti-adhesion extra thin Polyvinyl Alcohol foam membranes (ETPVAFM) could be established by determining morphology, water permeability, thickness, macro porosity, mechanical property of resulting samples.

\section{Methods}

An anti-adhesion Extra Thin Polyvinyl Alcohol Foam Membranes (ETPVAFM) for Minimally Invasive Surgery (MIS) was designed and prepared by using medical grade cenefom Polyvinyl Alcohol materials and a new super clean air-foaming process with active molecules such as 03. The PVA Foam membranes with fully opencell microstructures could be obtained. The anti-adhesion material 
was important for the clinic application of Minimally Invasive Surgery (MIS). Extra thin Polyvinyl Alcohol foam membranes (ETPVAFM) could be considered as a good anti-adhesion material for Minimally Invasive Surgery (MIS). An anti-adhesion extra thin foam membranes with good mechanical property would be a potential medical device for Minimally Invasive Surgery (MIS). A series of anti-adhesion PVAETF membranes with different macro porosities and thickness of 1.0 1.5 mm was designed and prepared such as ETPVAFM. Mechanical property of the resulting Extra Thin Foam Membranes (ETPVAFM) was determined.

\section{Results}

In this work, a novel anti-adhesion extra thin Polyvinyl Alcohol foam membranes (ETPVAFM) was obtained by using a clean air-foaming process, which could obtain foam matrix with partial open cell structure. The active molecules such as 03 were introduced into the super clean air-foaming process and fully open cell microstructures were easily obtained. The fully open cell microstructures could be observed by using OM. Furthermore, the structure could provide excellent water permeability, particularly, for MIS. The water permeability of the resulting anti-adhesion extra thin Polyvinyl Alcohol foam membranes (ETPVAFM) could be observed in the range of $82 \sim 85 \%$ by using ASTM D4491(standard test methods for water permeability). Averaged macroporosity values of the resulting extra thin Polyvinyl Alcohol (PVA) foam membranes were observed such as $475 \mu \mathrm{m}$ (Figure 1). The macroporosity could be changed depending on different clinical demands.

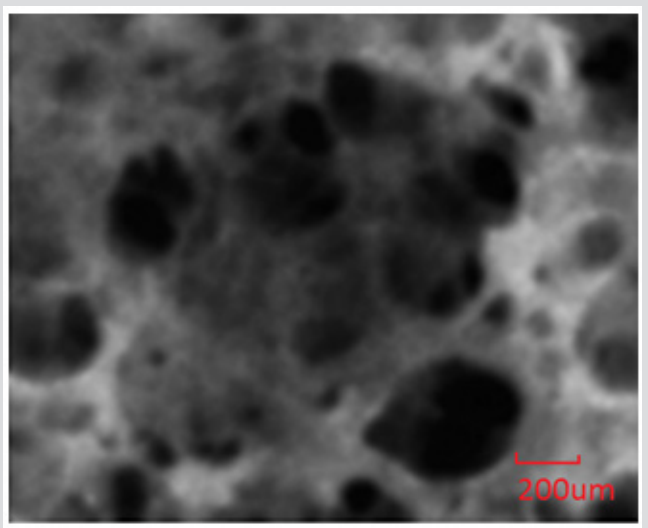

Figure 1: A new anti-adhesion extra thin polyvinyl alcohol (PVA) foam membranes with fully open cell microstructures.

Super clean air-foaming process was employed in the preparation of novel anti-adhesion extra thin Polyvinyl Alcohol (PVA) foam membranes with different macroporosities to obtain a kind of fully open cell microstructure, which could provide very short saturated water absorption time for MIS treatments. It spends a short-saturated water absorption time of $13 \mathrm{sec}$ from the dried state to the saturated water absorption state. Remarkable higher water uptake of the resulting extra thin Polyvinyl Alcohol (PVA) foam membranes than ca.1300 \% could be observed. The resulting anti-adhesion extra thin Polyvinyl Alcohol (PVA) foam membranes with high water contents $(>1300)$ still showed good elongation property such as ETPVAFM with $665.2 \%$ of elongation at break which could be considered as a kind of excellent materials for MIS. High permeability of foam dressings could be designed easily for polyurethane foam materials in the clinic application of negative pressure wound therapy (NPWT).

It is quite difficult to match the need for traditional Polyvinyl Alcohol (PVA) foam materials because of partial open-cell microstructure. However, polyurethane foam materials showed poor anti-adhesion property. For these reasons, the anti-adhesion extra thin Polyvinyl Alcohol (PVA) foam membranes could be considered as assistive anti-adhesion dressings with fully open-cell microstructure for negative pressure wound therapy (NPWT) to take care of ulcer (Figure 2).

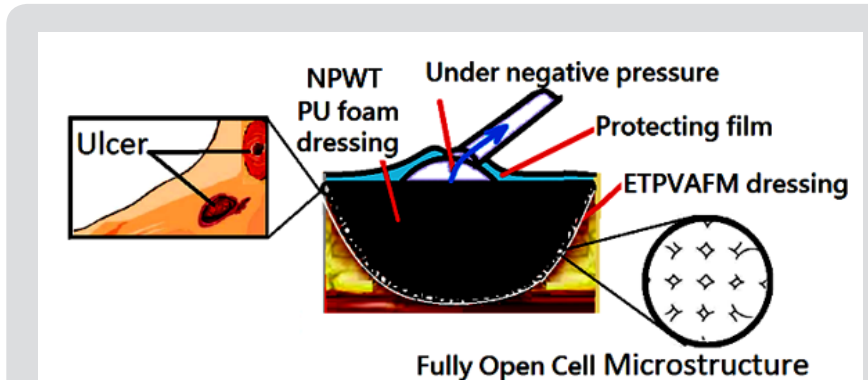

Figure 2: The anti-adhesion ETPVAFM could be considered as a kind of an assistive anti-adhesion dressing for negative pressure wound therapy (NPWT).

\section{Conclusion}

In this study, the anti-adhesion ETPVAFM with macroporosity of $475 \mu \mathrm{m}$ was obtained. The resulting anti-adhesion ETPVAFM with high water contents also showed good elongation property which could be considered as an excellent material for MIS. Furthermore, the resulting anti-adhesion ETPVAFM could provide a powerful potential in the clinic application of negative pressure wound therapy.

\section{Acknowledgement}

Authors would like to acknowledge the Taiwan PARSD Pharm. Tech. Ltd Co. for financial and technical supports. The authors also thank for technical assistances of C.-H. Shen.

\section{References}

1. Liaw DJ, Huang CC, Kang ET (2006) Effect of architecture and environments on polymeric molecular assemblies of novel amphiphilic diblock copolynorbornenes with narrow polydispersity via living ringopening metathesis polymerization (ROMP). Journal of Polymer Science Part A: Polymer Chemistry 44: 2901-2911.

2. Liaw DJ, Huang CC, Sang HC, Kang ET (1999) Photophysical and solution properties of naphthalene-labeled styrene/N, N-dimethyl maleimido propylammonium propane sulfonate copolymer. Langmuir 15: 52045211.

3. Zhai G, Toh SC, Tan WL, Kang ET, Neoh KG, et al. (2003) Poly (vinylidene fluoride) with grafted Zwitterionic polymer side chains for electrolyteresponsive microfiltration membranes. Langmuir 19: 7030-7037. 
4. Liaw DJ, Huang CC, Ju JY (2006) Novel star-like multifunctional polymeric materials with predominant cis microstructures derived from $\alpha$-norbornenyl macromonomer and stable macroinitiator via ring-opening metathesis polymerization and atom transfer radical polymerization. Journal of Polymer Science, Part A: Polymer Chemistry 44: 3382-3392.

5. Li ZF, Kang ET, Neoh KG, Tan KL, Huang CC, et al. (1997) Surface structures and adhesive-free adhesion characteristics of polyaniline films after modification by graft copolymerization. Macromolecules 30 3354-3362

ISSN: 2574-1241

DOI: 10.26717/BJSTR.2019.15.002672

Ching Cheng Huang. Biomed J Sci \& Tech Res

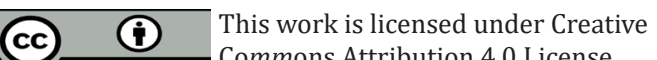

Submission Link: https://biomedres.us/submit-manuscript.php
6. Kang ET, Neoh KG, Huang CC, DJ Liaw, SC Toh, et al. (2003) Poly (vinylidene fluoride) with grafted Zwitterionic polymer side chains for electrolyte-responsive microfiltration membranes. Langmuir 19:70307037.

7. Huang CC (2018) A surgical anti-adhesion thin membrane Taiwan Pantent, M559718.

$\begin{array}{ll}\text { BIOMEDICAL } & \text { Assets of Publishing with us } \\ \text { RESEARCHES } & \text { - Global archiving of articles } \\ & \text { - Immediate, unrestricted online access } \\ & \text { - Rigorous Peer Review Process } \\ \end{array}$

